\title{
DISTÂNCIAS GENÉTICAS ENTRE TOUROS NELORE DE MATO GROSSO DO SUL, POR TÉCNICAS DE ANÁLISES MULTIVARIADA
}

\section{(Genetic distances between Nelore bulls from Mato Grosso do Sul, by multivariate analysis techniques)}

\author{
BELLO, A. B. S. ${ }^{1}$; FERRAZ FILHO, P. B. ${ }^{2}$; SILVA, L. O. C. ${ }^{3}$; SOUZA, J. C ${ }^{4}$; MUNIZ, N. M. ${ }^{5}$ \\ ${ }^{1}$ Pós Graduação em Ciência Animal - UFMS, Bolsista da FUNDECT \\ ${ }^{2}$ Departamento de Ciências Naturais - UFMS \\ ${ }^{3}$ Pesquisador Embrapa Gado de Corte - CNPGC \\ ${ }^{4}$ UFPR - Campus de Palotina \\ ${ }^{5}$ Pós Graduação em Ciência Animal - UFMS
}

\begin{abstract}
RESUMO - O objetivo deste estudo foi avaliar a divergência genética, entre reprodutores da raça $\mathrm{Ne}$ lore do Estado de Mato Grosso do Sul, com sêmens disponíveis em Centrais de Inseminação, com base nas diferenças esperadas em suas progênies com relação a caracteres ponderais e reprodutivos, através de técnicas de análises multivariadas. Medidas de dissimilaridade foram obtidas através das distâncias Euclidianas médias padronizadas, sendo a maior dissimilaridade entre os touros com distância de 3,4409 e a maior similaridade entre os progenitores com valor estimado de 0,3048 de distância. Três grupos de reprodutores com o mesmo padrão de similaridade foram obtidos pela técnica de agrupamento de Tocher. Escores dos componentes principais foram estimados, para se obter gráficos de dispersão, que possibilitaram a avaliação visual da divergência genética e do padrão de dissimilaridades entre os materiais estudados. Dos componentes principais, três deles explicaram $79,42 \%$ da variância total, o primeiro explicou $48,93 \%$, o segundo respondeu por $18,11 \%$ e o terceiro por $12,39 \%$ da variância. As estimativas de divergência genética encontradas permitem definir progenitores dissimilares que podem produzir ganhos significativos em função dos efeitos heteróticos oriundos de acasalamentos entre suas progênies.
\end{abstract}

Palavras-chave: análise multivariada; dissimilaridade; divergência genética; similaridade

\begin{abstract}
The objective of this study was to evaluate the genetic divergence, among sires of the race Nellore from Mato Grosso do Sul State, with available semen in head quarters Insemination, the evaluations were done using multivariate analysis between expected progeny differences of productive and reproductive traits. Dissimilarity measures were obtained through the Eclidian standardized averages distances, being the largest dissimilarity among the bulls (3.4409) and the largest similarity among the sires with approximate value of 0.3048 of distance. Three groups of reproducers with the same similarity pattern were obtained by the Tocher technique of grouping. Scores of principal components were estimates and the dispersion graphs were done. According to the principal components, three of them explained $79.43 \%$ of the total variances; the first explained $48.93 \%$, the second answered for $18.11 \%$ and the third for $12.39 \%$ of the variance. The estimates of genetic divergence give possibility to define sires dissimilarity that can produce significant gain in regard to of the heterotic effects originated from matings among their progenies.
\end{abstract}

Key words: Multivariate analysis; dissimilarity; genetic divergence; similarities 


\section{INTRODUÇÃO}

No melhoramento animal, procura-se obter material genético mais produtivo, e eficiente utilizando-se a seleção. Atualmente diversos grupos de pesquisadores têm desenvolvido pesquisas com modernos métodos de avaliação genética e de estimação de parâmetros genéticos para diversos critérios de seleção. Trabalhos envolvendo estimativas de parâmetros genéticos para diversas características, são reportados por MALHADO et al. (2002), GARCIA et al. (2003), GUIMARÃES et al. (2003), LIMA et al. (2005) e CONCEIÇÃO et al. (2005).

Medidas de dissimilaridade são importantes parâmetros utilizados pelos melhoristas principalmente quando, com base na divergência genética, se procura identificar genitores a serem utilizadas em programas que visem à obtenção de progênies com maior heterose. Estudos sobre a diversidade genética também têm sido realizados com o intuito de identificar genótipos com maior similaridade, para aumento de homozigose oriunda de endocruzamentos (CRUZ e REGAZZI, 1997).

Entretanto, são poucas as análises que abordam os efeitos da seleção sobre a endogamia na fixação de alelos. O conhecimento deste impacto é importante visto que a depressão endogâmica na característica sob seleção tem importante efeito sobre sua resposta a médio e em longo prazo (OLIVEIRA et al. 1999; SILVA et al., 2001).

A diversidade genética é um componente importante para a variabilidade populacional, podendo ser inferida por métodos preditivos tomando por base as diferenças morfológicas, fisiológicas ou moleculares (XAVIER, 2000). Recentemente, tem-se estudado a possibilidade de utilização de informações sobre similaridade e dissimilaridade genética, por intermédio de diferenças esperadas nas progênies (DEPs), utilizando-se de técnicas de análise multivariadas (FERRAZ FILHO et al., 2006).

Para muitos tipos de dados biológicos, há correlação entre variáveis, sendo que informações providas por análises univariadas podem ser incompletas. Assim, técnicas de análise multivariada combinam múltiplas informações provenientes de uma unidade experimental, que não são possíveis de serem obtidas com o uso da análise univariada (CRUZ e REGAZZI, 1997; XAVIER, 2000). Várias técnicas de análise multivariada podem ser aplicadas, entre as quais são citadas a análise de agrupamento e a análise de componentes principais.

O processo de agrupamento envolve, basicamente, duas etapas, a primeira relaciona-se com a estimação da similaridade, ou dissimilaridade entre progenitores com base na distância Euclidiana média e a segunda com a adoção de uma técnica de agrupamento para a formação dos grupos (PIASSI, 1994). Já a análise por meio de componentes principais, tem como objetivo a obtenção de um pequeno número de combinações lineares (componentes principais) de um conjunto de variáveis, que retenham o máximo possível da informação contida nas variáveis originais, eliminando as redundâncias (MACHADO, 1999).

Objetivou-se com este trabalho avaliar a diversidade genética por processos preditivos, com base nas DEPs para características produtivas e reprodutivas e realizar a formação de grupos similares de reprodutores e realizar a formação de grupos similares de reprodutores por técnicas de agrupamentos e de componentes principais, através de análises multivariadas.

\section{Material e Métodos}

Foram selecionados todos os 54 reprodutores da raça Nelore, pertencentes a fazendas localizadas no Estado de Mato Grosso do Sul, com sêmen disponível em Centrais de Inseminação do Brasil, relacionados no Sumário Nacional de Touros das Raças Zebuínas, Nelore 2005 (BRASIL, 2005), realizado pela Associação Brasileira de Criadores de Zebu (ABCZ) com parceria da Embrapa Gado de Corte. Utilizou-se informações das diferenças esperadas nas progênies (DEPs), estimadas para as seguintes características: Peso aos 120 dias de idade (efeito materno $=\mathrm{PM}$ e total materno $=\mathrm{TMM}$ ), Peso aos 240 dias de idade (efeito direto $=$ PD e total materno = TMD); Peso aos 420 dias de idade (efeito direto $=$ PS); Ganho de peso pré-desmama (efeito direto = GND, e total materno = TMGND); Ganho de peso pós-desmama (efeito direto $=$ GPD); Perímetro escrotal ao sobreano (efeito direto $=$ PES); Idade ao primeiro parto (efeito direto = IPP); Intervalo entre primeiro e segundo partos (efeito direto $=12 \mathrm{P}$ ) e Intervalo entre os demais partos (efeito direto $=\mathrm{IOP}$ ).

As medidas de dissimilaridade foram obtidas através das estimativas das distâncias Euclidiana média, baseadas nas doze variáveis acima descritas. $\mathrm{Na}$ análise de agrupamento dos reprodutores empregou-se o método de otimização de Tocher, o qual baseia-se nas referidas distâncias. Coeficientes de ponderação de componentes principais foram estimados, para se obter gráficos de dispersão, que possibilitaram a avaliação da divergência genética e do padrão de dissimilaridades entre os materiais estudados. Os dados foram processados pelo Programa Genes - Aplicativo Computacional em Genética e Estatística, versão Windows (CRUZ, 2001). 


\section{Resultados e Discussão}

As distâncias Euclidianas médias possibilitaram a identificação de reprodutores similares e dissimilares (TABELA1).

TABELA 1 - MEDIDAS DE DISSIMILARIDADE E DE SIMILARIDADE ENTRE TOUROS DA RAÇA NELORE, COM BASE NA DISTÂNCIA EUCLIDIANA MÉDIA

\begin{tabular}{|c|c|c|c|c|c|}
\hline Touro & RGD* & Máxima & $\begin{array}{l}\text { Touro mais } \\
\text { Dissimilar }\end{array}$ & Mínima & Touro mais Similar \\
\hline 1 & JOIA0881 & 2,4322 & 49 & 0,5218 & 10 \\
\hline 2 & HCAL0822 & 2,4403 & 49 & 0,5805 & 5 \\
\hline 3 & 19229 & 2,5440 & 40 & 0,4934 & 53 \\
\hline 4 & VRJ07768 & 2,5986 & 46 & 0,5594 & 20 \\
\hline 5 & 10989 & 2,0945 & 40 & 0,4629 & 15 \\
\hline 6 & J5159 & 2,6144 & 46 & 0,6185 & 26 \\
\hline 7 & E0839 & 2,4398 & 40 & 0,4913 & 23 \\
\hline 8 & RDA0040 & 2,1483 & 40 & 0,4510 & 27 \\
\hline 9 & AREA0042 & 2,0744 & 40 & 0,4018 & 35 \\
\hline 10 & STRO1229 & 2,1687 & 49 & 0,4719 & 33 \\
\hline 11 & G5675 & 2,2136 & 40 & 0,4901 & 33 \\
\hline 12 & F0941 & 2,1094 & 40 & 0,4610 & 50 \\
\hline 13 & MRA2274 & 2,5316 & 46 & 0,3309 & 45 \\
\hline 14 & G0274 & 2,6366 & 46 & 0,3309 & 45 \\
\hline 15 & BMF8437 & 2,0336 & 36 & 0,4629 & 5 \\
\hline 16 & F3522 & 2,6037 & 49 & 0,9320 & 24 \\
\hline 17 & KITO3266 & 2,2952 & 40 & 0,4096 & 28 \\
\hline 18 & BULL0310 & 2,8597 & 46 & 0,4327 & 37 \\
\hline 19 & J7166 & 2,5054 & 49 & 0,6395 & 39 \\
\hline 20 & GCMV2727 & 2,6939 & 46 & 0,4801 & 29 \\
\hline 21 & J5065 & 2,4124 & 46 & 0,5543 & 38 \\
\hline 22 & MRA2679 & 2,4826 & 40 & 0,3200 & 42 \\
\hline 23 & J5158 & 2,4699 & 49 & 0,4913 & 7 \\
\hline 24 & G8718 & 2,8710 & 49 & 0,9740 & 16 \\
\hline 25 & G9119 & 2,7531 & 40 & 0,7845 & 4 \\
\hline 26 & ARI1347 & 2,8371 & 40 & 0,5866 & 42 \\
\hline 27 & SEEN11408 & 2,0015 & 46 & 0,3230 & 33 \\
\hline 28 & JOIA0308 & 2,2640 & 40 & 0,4096 & 17 \\
\hline 29 & SEEN1676 & 2,5331 & 46 & 0,4694 & 20 \\
\hline 30 & G8719 & 2,7278 & 49 & 0,8396 & 7 \\
\hline 31 & 12349 & 3,1540 & 46 & 0,5643 & 34 \\
\hline 32 & JAN1765 & 2,2062 & 49 & 0,5070 & 33 \\
\hline 33 & PLF1225 & 2,0648 & 40 & 0,3230 & 27 \\
\hline 34 & ZAN0014 & 2,7201 & 46 & 0,5643 & 31 \\
\hline 35 & FBJ1646 & 1,9492 & 46 & 0,3240 & 27 \\
\hline 36 & J0077 & 3,0736 & 49 & 0,7770 & 19 \\
\hline 37 & PETB597 & 2,9164 & 46 & 0,4327 & 18 \\
\hline 38 & JDEAA0775 & 2,6052 & 46 & 0,5543 & 21 \\
\hline 39 & F8444 & 2,2488 & 49 & 0,6395 & 19 \\
\hline 40 & G1999 & 3,0833 & 49 & 1,9034 & 24 \\
\hline
\end{tabular}


TABELA 1 - MEDIDAS DE DISSIMILARIDADE E DE SIMILARIDADE ENTRE TOUROS DA RAÇA NELORE, COM BASE NA DISTÂNCIA EUCLIDIANA MÉDIA

\begin{tabular}{cccccc}
\hline Touro & RGD* & Máxima & $\begin{array}{c}\text { Touro mais } \\
\text { Dissimilar }\end{array}$ & Mínima & Touro mais Similar \\
\hline 41 & OTPO3793 & 2,2638 & 40 & 0,5573 & 9 \\
42 & JDEAA1140 & 2,6476 & 40 & 0,3200 & 22 \\
43 & TOTO8726 & 2,2358 & 49 & 0,4821 & 5 \\
44 & L2765 & 2,9301 & 36 & 1,0078 & 18 \\
45 & JOIA564 & 2,3422 & 46 & 0,3308 & 13 \\
46 & OTPO4216 & 3,4409 & 49 & 0,5620 & 51 \\
47 & F7477 & 2,7937 & 40 & 0,7028 & 42 \\
48 & G5228 & 2,4462 & 40 & 0,3048 & 53 \\
49 & CSCN6330 & 3,4409 & 46 & 0,9120 & 18 \\
50 & CSCN6454 & 2,1786 & 46 & 0,4609 & 12 \\
51 & TOTO9796 & 3,0225 & 49 & 0,5620 & 46 \\
52 & CSCN6634 & 2,6421 & 40 & 0,4694 & 29 \\
54 & EMMGG3758 & 2,4053 & 40 & 0,3048 & 48 \\
\hline
\end{tabular}

${ }^{*}$ RGD = Registro Geral Definitivo na Associação Brasileira de Criadores de Zebu (ABCZ)

Os resultados apresentados na TABELA 1 evidenciam os graus de similaridade e de dissimilaridade entre os touros avaliados. Estes mostraram que a maior dissimilaridade ocorreu entre os touros $46 \mathrm{e}$ 49 (distância Euclidiana média de 3,4409) e a maior similaridade entre os progenitores 48 e 53 (com valor estimado de 0,3048 de distância Euclidiana média).

Por intermédio das estimativas destas distâncias, é possível identificar entre os animais avaliados, aqueles mais divergentes. As progênies desses genótipos seriam recomendadas, em programas de melhoramento, como genitores nos acasalamentos, após uma análise criteriosa de seus desempenhos em relação a cada um dos caracteres avaliados, visando à heterose (CARNEIRO et al., 1999, CRUZ e CARNEIRO, 2003).

Como o número elevado de estimativas dificulta o reconhecimento de grupos homogêneos, pelo simples exame visual daquelas estimativas empregou-se o método de agrupamento de otimização (método de Tocher). $\mathrm{O}$ agrupamento dos touros por meio deste método, com base na dissimilaridade expressa pela distância Euclidiana média padronizada, permitiu o estabelecimento dos três grupos apresentados na TABELA 3, onde constatou-se que os touros 36, 46 e 40 foram os mais divergentes em relação aos 51 demais touros avaliados.

TABELA 2 - GRUPOS DE TOUROS DA RAÇA NELORE, ESTABELECIDOS PELO MÉTODO DE TOCHER, COM BASE NA DISSIMILARIDADE EXPRESSA PELA DISTÂNCIA EUCLIDIANA MÉDIA PADRONIZADA

\begin{tabular}{ll}
\hline Grupos & Touros \\
& $48,53,3,8,27,33,35,9,28,17,12,10,50,22,41,11,7,32,5,1,43,54,15,23,45$, \\
I & $42,29,21,52,4,38,20,13,6,26,14,25,39,37,18,2,47,19,34,30,16,44,31$, \\
& $51,24,49$ \\
II & 36,46 \\
III & 40 \\
\hline
\end{tabular}


Componentes principais também foram obtidos a partir das médias padronizadas dos 54 touros, avaliados em relação às diferenças esperadas em suas progênies (DEPs) para os doze caracteres apresentados. A matriz de correlação $(R)$, baseada nos dados originais, entre as variáveis, foi:

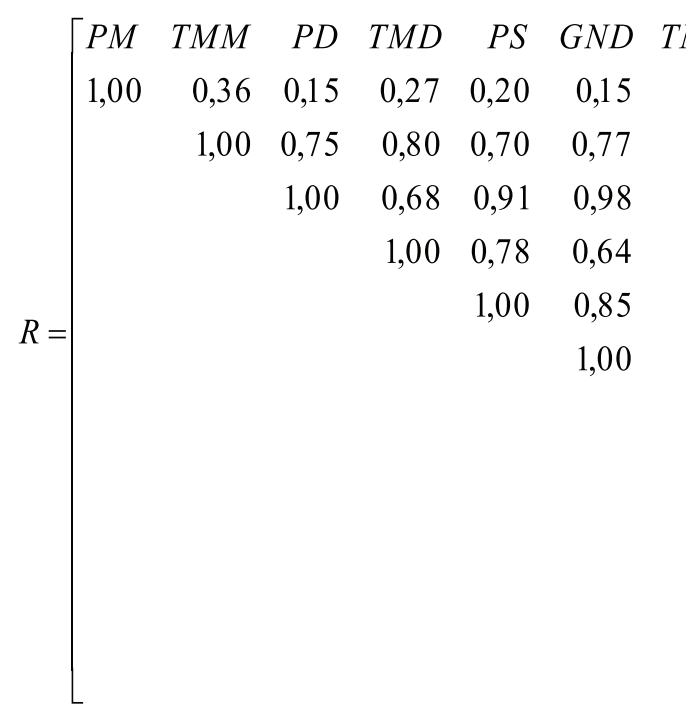

Obteve-se a matriz de covariância entre os valores padronizados, que corresponde à matriz de correlação dos dados expressos pelas médias originais, a partir da qual foi possível a estimativa dos autovalores e autovetores, apresentados nas TABELAS 3 e 4.

TABELA 3 - ESTIMATIVAS DE AUTOVALORES $\left(\lambda_{J}\right)$ OBTIDOS DA MATRIZ DE CORRELAÇÃO ENTRE AS DEPS ESTIMADAS

\begin{tabular}{ccc}
\hline$\lambda_{J}$ & $\lambda_{J_{\%}}$ & $\lambda_{J_{\%}}$ acumulada \\
\hline 5,87 & 48,93 & 48,93 \\
2,17 & 18,11 & 67,03 \\
1,49 & 12,39 & 79,42 \\
0,78 & 6,54 & 85,95 \\
0,56 & 4,83 & 90,78 \\
0.50 & 4,21 & 94,99 \\
0,25 & 2,04 & 97,03 \\
0,15 & 1,23 & 98,26 \\
0,11 & 0,91 & 99,17 \\
0,06 & 0,49 & 99,66 \\
0,03 & 0,27 & 99,93 \\
0,01 & 0,07 & 100,0 \\
\hline
\end{tabular}


Com os autovalores obtidos verificou-se serem necessários os três primeiros componentes principais para explicar $79,42 \%$ da variação entre os touros, conforme resultados apresentados na TABELA 3.

Os resultados evidenciam que os primeiros componentes explicam em torno de $80 \%$ da variação encontrada nos dados originais, sendo viável o estudo da divergência genética, por meio da dispersão em gráficos, cujas coordenadas são os escores relativos àqueles componentes principais (PIASSI, 1994; CRUZ e REGAZZI,1997). Neste caso, onde os quatro primeiros componentes principais explicaram mais de $80 \%$ da variação encontrada, optou-se pela dispersão gráfica tridimensional (GRÁFICO 1).

\section{FIGURA 1 - DISPERSÃO GRÁFICA DE 54 TOUROS DA RAÇA NELORE, EM RELAÇÃO AOS TRÊS PRIMEIROS COMPONENTES PRINCIPAIS}

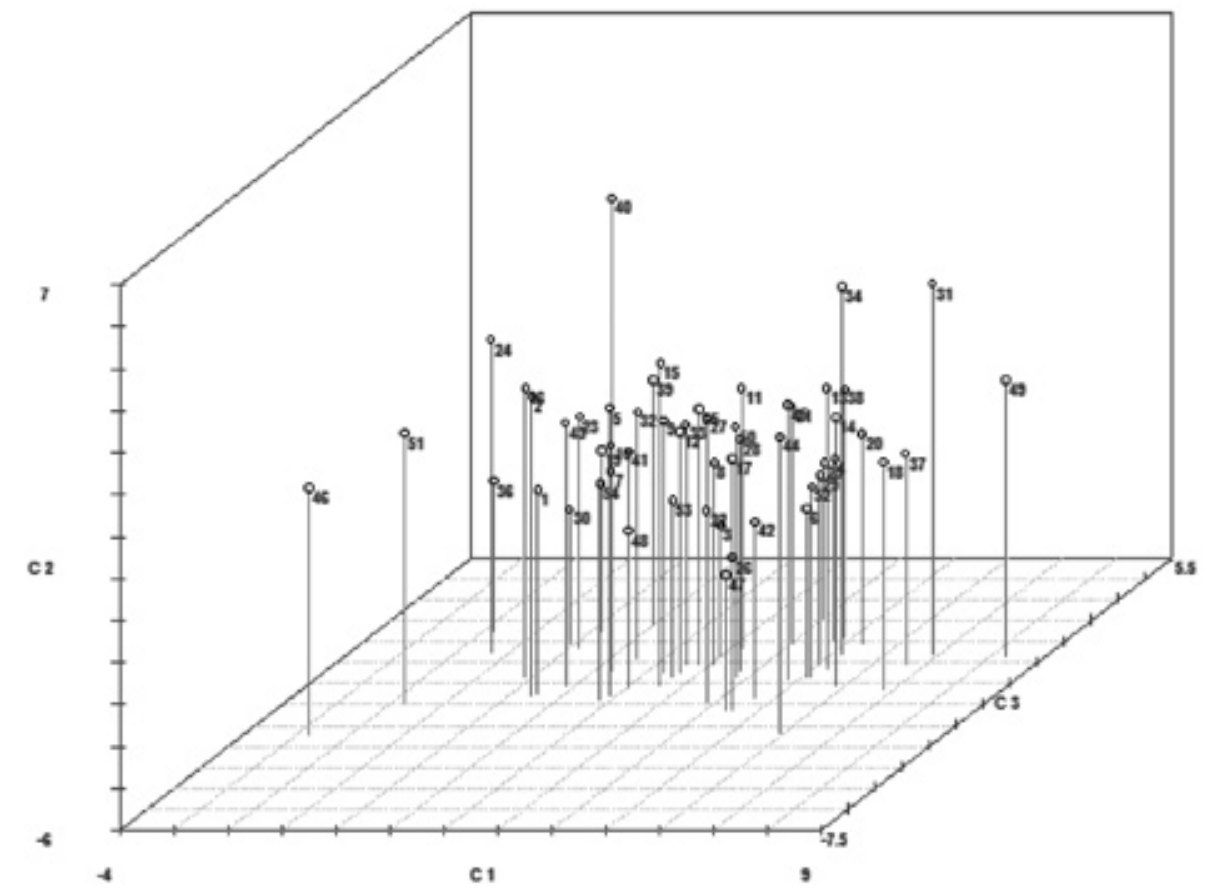

No gráfico tridimensional são considerados os escores dos três primeiros componentes principais, confirmando que os genótipos 46 e 49 apresentam a máxima diversidade por situarem-se graficamente mais distantes, já nos mais similares a visualização tornou-se difícil, por existir alta proximidade entre os touros que formam o grupo I. Este resultado permite confirmar a eficiência do método de componentes principais, onde apenas os três primeiros foram suficientes para representar a formação dos grupos.
A dispersão gráfica resultante dos componentes principais deverá ser utilizada tanto na identificação de cruzamentos promissores quanto aqueles cuja variabilidade disponível em gerações segregantes deve ser restrita (CRUZ e REGAZZI, 1997).

A estimativa dos componentes principais permitiu avaliar ainda, por intermédio dos autovetores (Tabela 4), a importância relativa das características empregadas sobre a diversidade genética 
TABELA 4 - CONJUNTO DE AUTOVETORES OBTIDOS DA MATRIZ DE CORRELAÇÃO ENTRE AS VARIÀVEIS

\begin{tabular}{|c|c|c|c|c|c|c|c|c|c|c|c|c|}
\hline \multirow[b]{2}{*}{ CP } & \multicolumn{12}{|c|}{ Variável Original } \\
\hline & PM & TMM & PD & TMD & PS & GND & TMGD & GPD & IPP & I2P & IOP & PES \\
\hline $1^{a}$ & $-0,13$ & 0,36 & 0,38 & 0,34 & 0,38 & 0,38 & 0,36 & 0,31 & $-0,16$ & $-0,74$ & 0,046 & 0,19 \\
\hline $2^{\mathrm{a}}$ & $-0,24$ & 0,02 & 0,04 & 0,14 & 0,08 & 0,01 & 0,05 & 0,12 & 0,53 & 0,53 & 0,49 & $-0,05$ \\
\hline $3^{\mathrm{a}}$ & $-0,40$ & $-0,26$ & 0,24 & $-0,27$ & 0,15 & 0,23 & $-0,29$ & 0,05 & 0,14 & $-0,71$ & $-0,15$ & 0,66 \\
\hline $4^{a}$ & 0,73 & $-0,15$ & $-0,03$ & $-0,14$ & 0,03 & $-0,06$ & $-0,21$ & 0,13 & 0,13 & 0,15 & 0,47 & $-0,32$ \\
\hline $5^{a}$ & $-0,24$ & $-0,23$ & $-0,18$ & 0,13 & 0,24 & $-0,26$ & $-0,17$ & 0,77 & 0,12 & $-0,82$ & $-0,28$ & $-0,18$ \\
\hline $6^{a}$ & $-0,46$ & $-0,06$ & $-0,12$ & 0,02 & $-0,04$ & $-0,11$ & $-0,01$ & 0,17 & $-0,40$ & $-0,38$ & $-0,65$ & $-0,02$ \\
\hline $7^{\mathrm{a}}$ & 0.07 & $-0,38$ & 0,08 & $-0,40$ & 0,37 & $-0,18$ & $-0,26$ & $-0,26$ & $-0,48$ & 0,38 & 0,01 & 0,06 \\
\hline $8^{\mathrm{a}}$ & 0,05 & 0,20 & 0,13 & $-0,66$ & 0,06 & 0,07 & $-0,11$ & 0,27 & $-0,43$ & 0,45 & 0,04 & $-0,16$ \\
\hline $9^{\mathrm{a}}$ & $-0,01$ & 0,15 & $-0,37$ & 0,13 & $-0,36$ & $-0,28$ & 0,30 & 0,19 & $-0,20$ & 0,31 & $-0,11$ & 0,59 \\
\hline $10^{\mathrm{a}}$ & 0,08 & $-0,71$ & 0,04 & $-0,07$ & $-0,24$ & 0,37 & 0,49 & 0,12 & $-0,09$ & 0,08 & 0,00 & $-0,11$ \\
\hline $11^{a}$ & $-0,02$ & $-0,09$ & $-0,09$ & $-0,35$ & 0,58 & $-0,42$ & 0,54 & $-0,20$ & 0,11 & $-0,09$ & $-0,00$ & 0,07 \\
\hline $12^{\mathrm{a}}$ & 0,00 & 0,06 & $-0,76$ & $-0,02$ & 0,33 & 0,54 & $-0,08$ & -0.08 & $-0,02$ & 0,04 & 0,01 & 0,11 \\
\hline
\end{tabular}

$\mathrm{CP}=$ Componente Principal, $\mathrm{PM}=$ Peso aos 120 dias de idade (efeito materno); $\mathrm{TMM}=\mathrm{Peso}$ aos 120 dias de idade (total materno) PD= Peso aos 240 dias de idade (efeito direto = PD); TMD = Peso aos 240 dias de idade (total materno); PS= Peso aos 420 dias de idade (efeito direto); GND= Ganho de peso pré-desmama (efeito direto); TMGD= Ganho de peso pré-desmama (total materno); GPD = Ganho de peso pós-desmama (efeito direto); IPP = Idade ao primeiro parto (efeito direto); I2P = Intervalo entre primeiro e segundo partos (efeito direto); IOP = Intervalo entre os demais partos (efeito direto); PES = Perímetro escrotal ao sobreano (efeito direto).

Considera-se que variáveis com pequena variabilidade ou que estão correlacionadas com outras consideradas no estudo, apresentarão coeficientes de grande magnitude nos últimos autovetores, a importância decresce da última à primeira função linear (CRUZ e REGAZZI, 2001; CRUZ e CARNEIRO, 2003).

Após análise dos componentes cujo autovalor, obtido da matriz de correlação, foram identificadas, em ordem crescentes, as variáveis de maior coe- ficiente na última função linear. Segundo CRUZ e REGAZZI (1997), o descarte das características de maior coeficiente de ponderação pode ser feito a partir do último componente principal até aquele que não exceda ao número índice 0,70 e identificando para descarte, a variável cujo coeficiente no autovetor for o maior (valor absoluto). Adotando-se este critério, concluiu-se que as características de menor importância, passíveis de descarte foram: PD, PS, TMM, PES, TMD, IPP, IOP, I2P e PM. 


\section{CONCLUSÕES}

Os processos foram eficientes na identificação de distâncias genéticas, permitindo a formação de grupos de similaridade por métodos de otimização e os componentes principais permitiram a observação visual da divergência entre os reprodutores.

\section{AGRADECIMENTOS}

À FUNDECT/CAPES, pelo apoio financeiro.

\section{REFERÊNCIAS}

BRASIL. Ministério da Agricultura, Pecuária e do Abastecimento. Sumário Nacional de Touros das Raças Zebuínas: Nelore, edição 2005/MAPA. Embrapa Gado de Corte/ABCZ, 2005.

CARNEIRO, P.L.S.; EUCLYDES, R.F.; SILVA, M.A.E. et al. Efeito da seleção errônea de machos sobre ganhos genéticos, utilizando-se simulação. Revista Brasileira de Zootecnia, v.28, p.264-268, 1999.

CONCEIÇÃO, F.M., FERRAZ FILHO, P.B., SILVA, L.O.C., BRAGANÇA, V.L.C., SOUZA, J.C. Fatores ambientais que influenciam o peso à desmama, ano e sobreano em bovinos da raça Nelore Mocha, no sudoeste de Mato Grosso do Sul - Brasil. Archives of Veterinary Science v. 10, n. 2, p. 157-165, 2005.

CRUZ, C.D. Programa Genes: versão Windows; aplicativo computacional em genética e estatística. Viçosa: UFV, 2001. 648p.

CRUZ, C.D. Princípios de Genética Quantitativa. Viçosa: UFV, 2005. 394p.

CRUZ, C.D.; CARNEIRO, P.C.S. Modelos Biométricos Aplicados ao Melhoramento Genético. v. 2. Viçosa: UFV, 2003. 585 p.

CRUZ, C.D.; REGAZZI A.J. Modelos biométricos aplicados ao melhoramento genético. Viçosa, MG: UFV, 1997. 390p.

CRUZ, C.D.; REGAZZI, A.J. Modelos biométricos aplicados ao melhoramento genético. 2 ed. Viçosa: Imprensa Universitária, 2001. 390 p.
FERRAZ FILHO, P.B.; SILVA, L.O.C.; SOUZA, J.C.; MALHADO, C.H.M. Divergência genética de touros Nelores com sêmen disponível em centrais de inseminação no Brasil . In: REUNIÃO ANUAL DA SOCIEDADE BRASILEIRA DE ZOOTECNIA, 43. João Pessoa. Anais... João Pessoa: SBZ, 2006

GARCIA, F.Q., FERRAZ FILHO, P.B., SOUZA, J.C., SILVA, L.O.C. Tendência dos efeitos genéticos diretos e maternos do peso a desmama de bovinos da raça Nelore mocha na região pecuária Campo Grande e Dourados - Mato Grosso do Sul. Archives of Veterinary Science v. 8, n. 1, p. 93-97, 2003

GUIMARÃES, L.B., FERRAZ FILHO, P.B., SOUZA, J.C., SILVA, L.O.C. Aspectos genéticos e de ambiente sobre pesos pré e pós desmama em bovinos da raça Tabapuã na região pecuária oeste São Paulo - Paraná. Archives of Veterinary Science v. 8, n. 1, p. 109-119, 2003

LIMA, A.E.S., FERRAZ FILHO, P.B., SILVA, L.O.C., SOUZA, J.C., GONDO, A. Efeitos genéticos diretos e maternos e suas tendências em pesos à desmama de bovinos da raça Nelore Mocha, na região pecuária de Goiás. Archives of Veterinary Science v. 10, n. 2, p. 69-74, 2005

MACHADO, C. F. Procedimentos para a escolha de genitores de feijão. Lavras : UFLA, 1999. 118 p. Dissertação de Mestrado.

MALHADO, C.H.M., SOUZA, J.C., SILVA, L.O.C., FERRAZ FILHO, P.B. Correlações genéticas, fenotípicas e de ambiente entre os pesos de várias idades em bovinos da raça Guzerá no estado de São Paulo. Archives of Veterinary Science v.7, n.1, p.71-75, 2002

MORRISON, D.F. Multivariate Statistical Methods, 2.ed. New York: McGraw-Hill Book Company, 1976. $415 p$.

OLIVEIRA, J.A.; BASTOS, J.F.P.; TONHATI, H. Endogamia em um rebanho da raça Guzerá. Revista Brasileira de Zootecnia, v.28, n.4, p.721-728, 1999.

PIASSI, M.A. Avaliação do desempenho de linhagens de postura mantidas na Universidade Federal de Viçosa, em competição com marcas comerciais. Viçosa, MG: UFV, 1994, 86p. Dissertação (Mestrado em Genética e Melhoramento) - Universidade Federal de Viçosa, 1994. 
SILVA, M.V.G.B.; FERREIRA, W.J.; COBUCI, J.A. et al. Efeito da endogamia sobre características produtivas e reprodutivas de bovinos do ecótipo Mantiqueira. Revista Brasileira de Zootecnia, v.30, n.4, p.1236-1242, 2001.

XAVIER, L.H. Modelos univariado e multivariado para análise de medidas repetidas e verificação da acurácia do modelo univariado por meio de simulação. Piracicaba: Escola Superior de Agricultura "Luiz de Queiroz", 2000. 91p. Tese (Doutorado em Estatística e Experimentação Agronômica) - Escola Superior de Agricultura “Luiz de Queiroz”, 2000.

Recebido para publicação:

$12 / 12 / 2007$

Aprovado:

$05 / 05 / 2008$ 